\title{
Influence of the Type of Sea Waves on the Backscattered Radar Cross Section at Medium Incidence Angles
}

\author{
V. Yu. Karaev ${ }^{a, *}$, M. A. Panfilova ${ }^{a}$, and Guo Jie ${ }^{b}$ \\ ${ }^{a}$ Institute of Applied Physics Russian Academy of Sciences, Nizhny Novgorod, Russia \\ ${ }^{b}$ Yantai Institute of Coastal Zone Research, Chinese Academy of Sciences, Yantai, China \\ *e-mail:volody@hydro.appl.sci-nnov.ru \\ Received September 12, 2014
}

\begin{abstract}
We consider the influence of the sea surface state on the backscattered radar cross section and the accuracy of the wind speed retrieval from the scatterometer data. We used a joint set of radars and buoys to determine the type of sea waves. Three types of sea waves were distinguished: developing wind waves, fully developed wind waves, and mixed sea. It is shown that the retrieval error of the near surface wind speed using a one-parameter algorithm is minimal in the case of fully developed wind waves. We compared these data with the results of radio-altimeter data analysis and showed that in both cases underestimation of the retrieval wind speed exists for developing wind waves and overestimation occurs for mixed sea. A variety of swell parameters (length of the dominating wave, swell height, swell age) significantly influence the backscattered radar cross section, leading to a growth in the mean square error of the retrieved wind speed during vertical sounding (radio-altimeter data), and only slightly influence the mean square error of the scatterometer data (medium incidence angles). It is necessary to include the information about the parameters of sea waves in the algorithms and take into account the regional wave properties to increase the accuracy of wind speed retrieval.
\end{abstract}

Keywords: sea waves, scatterometer, backscattered radar cross section, wind speed, wave age, classification of sea waves, wind speed retrieval algorithms

DOI: $10.1134 /$ S0001433816090139

\section{INTRODUCTION}

Microwave radars are widely used to determine the wind speed and direction over the sea surface. Scatterometers are the main satellite-borne radars, which retrieve the surface wind field. Owing to a wide swath, one scatterometer can collect data from $90 \%$ of the World Ocean surface in one day (for example, Halpern, 2000).

According to the definition, scatterometers are radars designed for accurate measurements of the backscattered signal, but it is not possible to use them for the direct measurements of wind speed. Scattering of electromagnetic waves also occurs at the sea surface; during the processing of reflected radar signals, we calculate the backscattered radar cross section, which contains information about the parameters of the scattering surface depending also on the wind speed.

Satellite scatterometers operate in the medium range of incident angles when the backscattering mechanism is a resonance one. Scattering in the microwave range occurs at small resonance ripples (for example, Bass and Fuks, 1972; Valenzuela, 1978), and the backscattered radar cross section is proportional to the spectral density of ripples. The latter is sensitive to the surface wind speed; therefore, wind speed can be estimated from the variations in the backscattered radar cross section.

In the general case, the relation between wind speed and parameters of wind waves is not unambiguous; this leads to the errors in retrieving the wind speed using one-parameter algorithms. Unambiguous correlation between the backscattered radar cross section and wind speed is assumed if one-parameter algorithms are applied: the greater the backscattered radar cross section measured by a scatterometer, the greater the wind speed.

Since formation of sea waves not only occurs under the influence of the local wind but also depends on a number of other factors, it is interesting to distinguish different wave types and estimate their correlation with the backscattered radar cross section.

It is known that characteristics of wind waves strongly depend of the fetch; in particular, the significant wave height increases with the increase in the fetch, while the variance of the slopes of large-scale waves decreases. As a result, the variations in the backscattered radar cross section in the course of wave development over the fetch would be related to the variations 
in the degree of the development of wind waves (wave spectrum) provided that the wind speed is constant.

Wind waves over the fetch can gain the stage of developed waves and no further variations of the parameters of wind waves would occur as the fetch increases (Davidan et al., 1974). The correlations between the fully developed wind waves and backscattered radar cross section is unambiguous.

Swell significantly influences the formation of wind waves. Swell is formed in the region of strong winds, for example, hurricanes. Swell is capable of spreading over hundreds and thousands of kilometers from the place of formation. As a result, the characteristics of swell are not related to the local wind and significantly change the state of waves at the location of measurements. This leads to unambiguous correlation between wind speed and backscattered radar cross section, which will result in errors of wind speed and direction retrieval using the standard one-parameter algorithm.

The first investigations of the influence of wave type on the backscattered radar cross section and the accuracy of retrieving wind speed were conducted in the 1980s (for example, Glazman, 1987; Glazman and Pilorz, 1989, 1990). The first classification of wave types was proposed, and it was shown that the backscattered radar cross section during nadir sounding (radio altimeter) and consequently the retrieved wind speed depend on the type of waves. Accounting for this effect in the algorithm made it possible to increase the accuracy of wind speed retrieval from the Geosat radio-altimeter data (Glazman and Pilorz, 1990).

In our works, we also studied the problem of wave type classification and their influence on the accuracy of surface wind retrieval using radio altimeters (Karaev et al., 2006, 2013). In this case, backscattering is quasispecular; it occurs in the regions of a wave profile oriented normal to the incident irradiance.

Our Chinese colleagues collected a similar database for the ERS-1 and ERS-2 scatterometers (hereinafter, ERS-1/2); therefore, the possibility appeared to perform a similar analysis for scatterometers (medium incident angles) and compare the data with the results obtained using a radio altimeter (vertical sounding).

\section{CLASSIFICATION OF WAVE TYPES}

First of all, we give brief information about the classification of wave types used in the analysis of radioaltimeter data (vertical sounding) in (Karaev et al., 2006).

A radio altimeter measures the backscattered radar cross section and the height of significant waves; hence, investigators obtain reliable information about one of the wave characteristics. Application of this information made it possible to develop more precise two-parameter algorithms for retrieving wind speed from radio-altimeter data (Karaev et al., 2002; Gour- rion et al., 2002). The backscattered radar cross section and significant wave height are the input parameters for the new algorithms.

A simple classification of waves was proposed in (Karaev et al., 2002), which included two types. Developing wind waves and mixed sea are the two extreme wave types. The fully developed wind wave type is located between them when unambiguous correspondence exists between wind speed and significant wave height, which does not depend on the region. This relation can be plotted on the plane "wind speed-significant wave height" in the form of a curve (equilibrium curve).

Let us assume that, if a point on the plane "wind speed-significant wave height" is located below the curve, then developing waves exist at the sea surface, and if the point is below the curve, then the wave type is mixed sea.

In this work we assume that mixed sea are two-mode waves and the swell parameters are not related to the local wind speed. We introduce the following criterion to divide mixed sea into a number of domains: differential height $\Delta H_{S}$, which is the difference between the height of surface waves measured by the radio altimeter $H_{S}$ and the height of fully developed wind waves $H_{S f d}$ from the data of wind speed $U_{10}$ measured by a wave buoy. The curves located above the equilibrium curve (index 1) in Fig. 1 show the regions with equal swell height. The step of the swell height was assumed equal to $0.5 \mathrm{~m}$ (Karaev et al., 2002).

Generally speaking, this approach is not absolutely correct because we do not know the degree of wind wave development; hence, we do not know the actual height of wind waves. However, this problem can be solved without analysis of the wave spectrum, which is not available at this stage. We note that the corresponding error would be minimal at low wind speed but can gain high values at strong wind when significant time and wave fetch are needed.

Another division criterion was selected for the developing waves related to the fetch. We consider that the fetch is proportional to the wave heights and specify the boundaries of the interval as follows:

$$
\left[0.9 H_{S f d} ; H_{S f d}\left[, \quad\left[0,8 H_{S f d} ; 0,9 H_{S f d}[.\right.\right.\right.
$$

The boundaries of the regions of developing waves are shown in Fig. 1 with the curves located below the equilibrium curve. Asterisks show the data from the joint database of radio-altimeter data and the data of the NDBC buoys (Karaev et al., 2002).

\section{INITIAL DATA}

The ERS satellite missions consisted of two research satellites for remote sensing of the Earth launched by the European Space Agency in 1991 (ERS-1) and 1995 


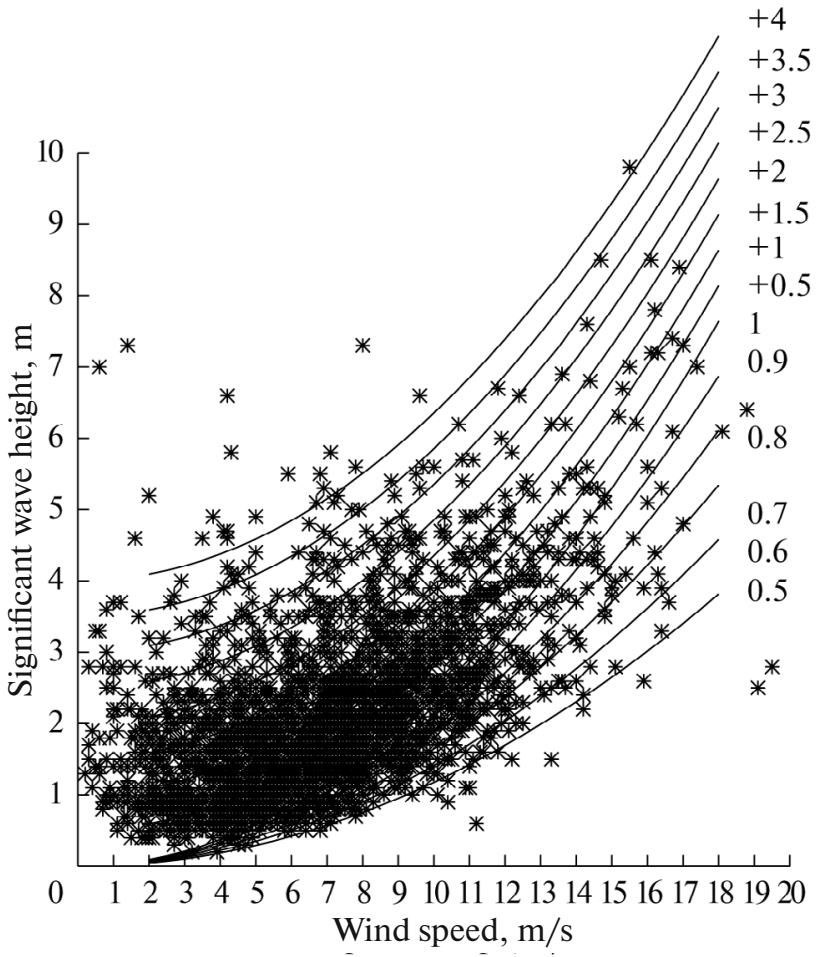

Fig. 1. Significant wave height vs. wind speed. The curve with index 1 corresponds to fully developed wind waves (equilibrium curve). The curves show the boundaries between the regions of mixed sea (above the equilibrium curve) and developing wind waves (below the equilibrium curve) (Karaev et al., 2002).

(ERS-2). Scatterometers were included in the research equipment installed on these satellites.

We study the influence of the wave type on the accuracy of wind speed retrieval based on the scatterometer data from the ERS-1/2 scatterometers (backscattered radar cross section and retrieved wind speed) and the data of marine wave NDBC buoys (significant wave height, wind speed and direction). The wind speed was retrieved using the standard one-parameter algorithm.

As a result of preliminary processing, we formed a joint database of the radar and moored buoy data covering a period from 1991 to 2001 (Guo Jie, 2009). We used the following conditions as the criteria:

(1) The distance between the moored buoy and the cell, in which the backscattered radar cross section is measured, does not exceed $0.15^{\circ}$.

(2) The time interval between the radar and buoy measurements does not exceed $30 \mathrm{~min}$.

Preliminary processing filtered out the measurements made during rain, when the power of the reflected signal is strongly attenuated.

As a result, each element of the joint dataset contained the following information: (1) wind speed and direction measured on the NDBC buoys; (2) signifi- cant wave height measured on buoys; (3) backscattered radar cross section and incident angle; (4) direction of the satellite track and direction of sounding; (5) wind speed retrieved from the scatterometer data.

Points with wind speed exceeding $3 \mathrm{~m} / \mathrm{s}$ were selected for further analysis. Wind waves are not formed if wind speed is lower (Donelan, Pierson, 1987); hence, wind does not influence the characteristics of surface waves and it cannot be retrieved from the backscattered radar cross section.

The final joint dataset of scatterometer and buoy data contains 3447 points (wind vector cells). A similar description of the joint dataset of radio-altimeter and buoy data is given in (Guo Jie, 2009).

Radio altimeters perform measurements during vertical sounding; therefore, it is easy to plot the dependence of the backscattered radar cross section on wind speed because there is no dependence of the backscattered radar cross section on the wind direction.

The ERS-1/2 scatterometer performs measurements in one wind cell at three azimuth angles; the incident angles are also different. Hence, construction of the dependence of the backscattered radar cross section on wind speed becomes more difficult. The dataset should be divided into parts according to the incident angle $\left(30^{\circ}-60^{\circ}\right)$ and azimuth angle $\left(0^{\circ}-\right.$ $360^{\circ}$ ). As a result, we obtain a small number of points for each "combination" and they cannot be used for the analysis.

A geophysical model function (GMF) is used to retrieve the wind speed. Three backscattered radar cross sections measured at different azimuth angles and incident angles in one wind cell are used as the input parameters of the algorithm. The measured backscattered radar cross sections contain all errors of measurements; therefore using the GMF and retrieved wind speed as input parameters, we can calculate the backscattered radar cross section for arbitrary incident and azimuth angles. This backscattered radar cross section will take into account all errors (atmospheric and instrumental noises, etc.), which existed in the initial data.

As a result, we calculated the backscattered radar cross sections for the entire dataset (3447 points) for the incident angle equal to $45^{\circ}$ and azimuth angle equal to $0^{\circ}$ (wind direction related to the direction of sounding).

Figure 2 shows the dependence of the backscattered radar cross section on wind speed for a radio altimeter (a) and scatterometer (b). Only wind speed exceeding $3 \mathrm{~m} / \mathrm{s}$ were used for this plot.

It is seen from the figure that the backscattered radar cross section decreases with increasing wind speed at zero incident angles and increases at moderate incident angles. This is related to the difference in the mechanisms of backscattering. 
(a)

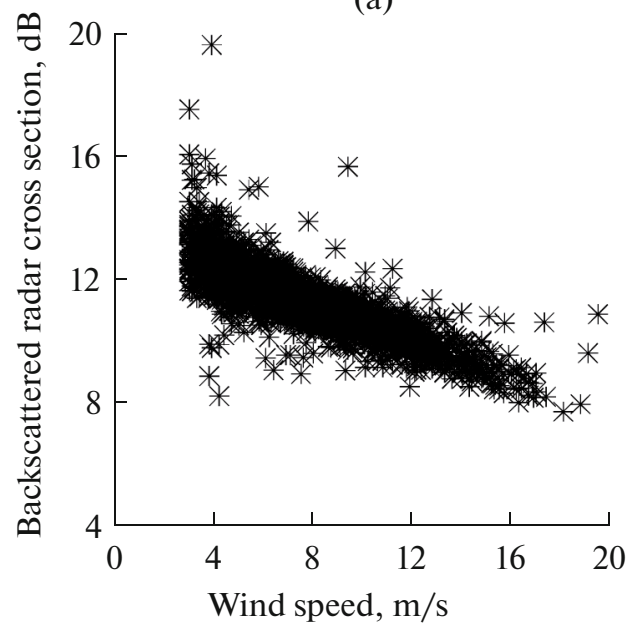

(b)

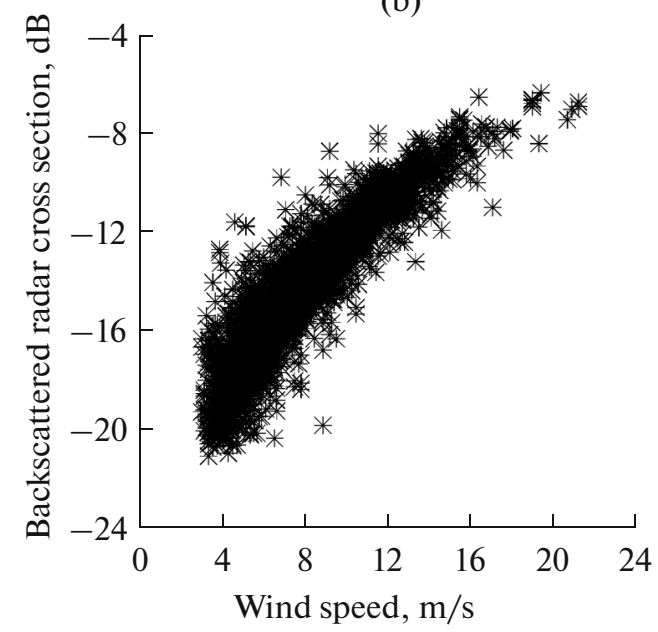

Fig. 2. Backscattered radar cross section vs. wind speed based on the Topex/Poseidon radio-altimeter data (a) and the ERS-1/2 scatterometers (b) at incident angles of $45^{\circ}$ and wind directed to the radar.

Scattering of the values seen in the figure is caused by ambiguity in the correlation between the backscattered radar cross section and wind speed. Processing of such data using the standard one-parameter algorithm leads to errors in wind speed retrieval. The error of wind speed retrieval is proportional to the width of the band (dispersion of data scattering).

\section{INFLUENCE OF THE WAVE TYPE ON THE ACCURACY OF WIND SPEED RETRIEVAL}

The data of sea buoys on wind speed $U_{10}$ were used while developing the algorithms for wind speed retrieval from the radar data. Since radio altimeters measure significant wave height independently, it is possible to perform classification of wave types on the basis of radio-altimeter data (backscattered radar cross section, significant wave height) and use this information in the data processing. Two-parameter algorithms for retrieving wind speed were developed using this fact, which are more accurate than one-parameter algorithms (Karaev et al., 2002; Gourrion et al., 2002).

Scatterometers measure only the backscattered radar cross section; therefore, it is not possible to perform classification of wave types and the "universal" one-parameter algorithm is used to retrieve wind speed.

Accuracy of wind speed retrieval from the ERS-1/2 scatterometer data

\begin{tabular}{l|c|c}
\hline \multicolumn{1}{c|}{ Waves } & $\mathrm{M}, \mathrm{m} / \mathrm{s}$ & $\mathrm{RMS}, \mathrm{m} / \mathrm{s}$ \\
\hline Developing wind waves & -0.71 & 1.17 \\
Fully developed wind waves & -0.61 & 0.98 \\
Mixed sea & -0.18 & 1.09 \\
\hline
\end{tabular}

However, as a result of formation of a joint dataset, which includes the radar measurements and data of sea buoys, there appears the possibility to perform classification of wave types and investigate the influence of wave type on the accuracy of wind speed retrieval using the universal scatterometer algorithm and compare it with the results of radio-altimeter data analysis.

Let us introduce the errors of wind speed measurements $\Delta U_{10}$ as follows:

$$
\Delta U_{10}=U_{10 \mathrm{r}}-U_{10},
$$

where $U_{10 \mathrm{r}}$ is the wind speed retrieved using the algorithm and $U_{10}$ is the wind speed measured on a buoy. As a result, the wind speed based on the scatterometer data was retrieved with a root-mean-square (RMS) deviation equal to $1.12 \mathrm{~m} / \mathrm{s}$ and mean value $M=-0.36 \mathrm{~m}$.

Let us estimate the accuracies of wind speed retrieval using the scatterometer data for different wave types according to the procedure used for radio altimeters. According to the classification of wave types, let us separate the developing waves and mixed sea. The data on the accuracies of different algorithms retrieving the wind speed based on the scatterometer data with consideration for the wave type are given in the table.

The waves were considered fully developed if differential height $\Delta H_{S}$ was in the interval from 0 to $0.25 \mathrm{~m}$.

As was expected, the dispersion is minimal for fully developed waves.

We continue separation within each type of waves for each interval according to Fig. 1 and calculate the mean deviation $M$ and the root-mean-square error (RMS) of wind speed retrieval from the scatterometer data. The results of radio-altimeter data processing were taken from (Karaev et al., 2006). 
(a)

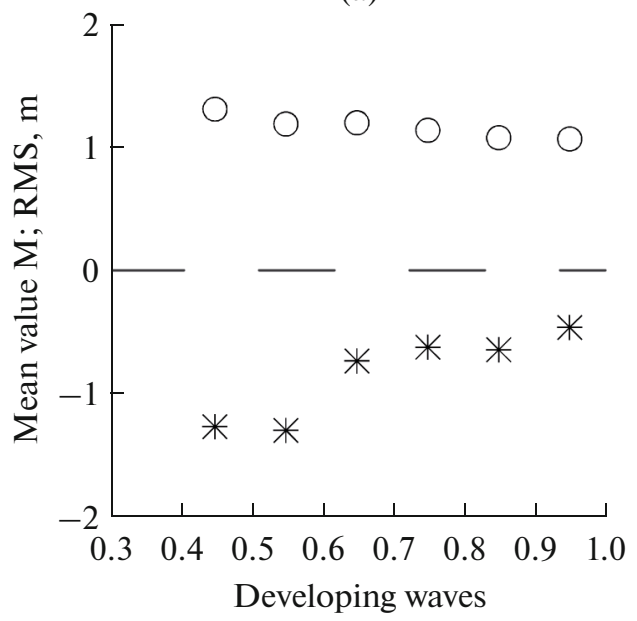

(b)

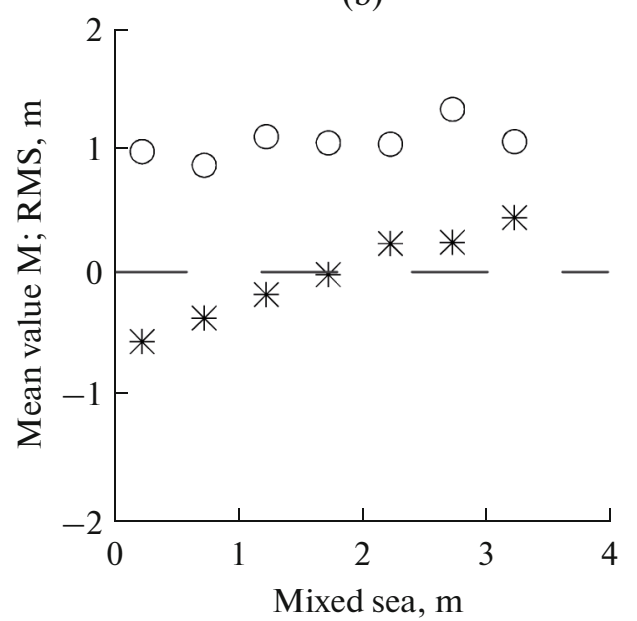

Fig. 3. Dependence of mean (asterisks, $M$ ) and root-mean-square deviation (circles, RMS) on the degree of development of wind waves (a) and height differences $\Delta H_{S}$ (b) for the ERS-1/2 scatterometers.

(a)

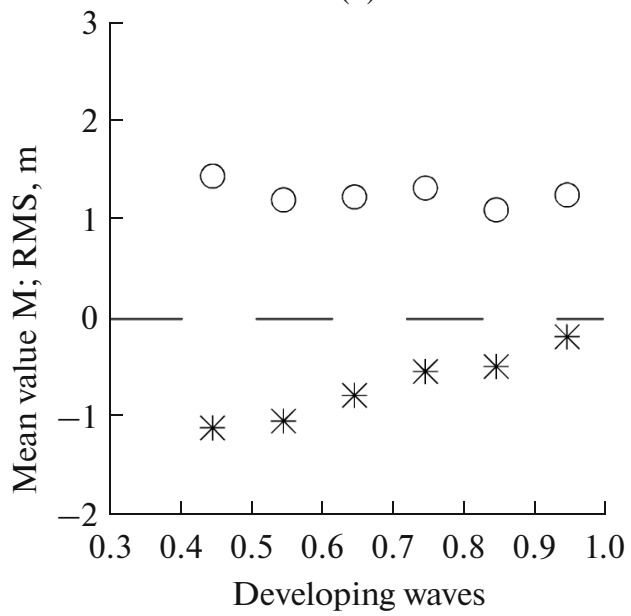

(b)

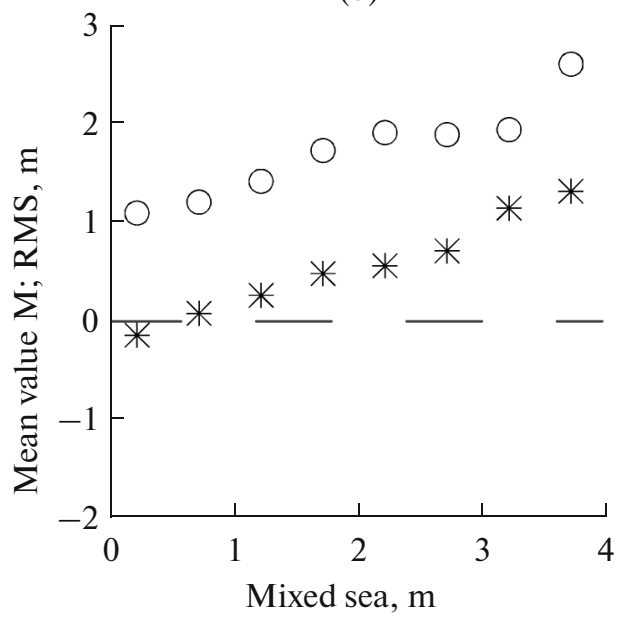

Fig. 4. Dependence of mean (asterisks, $M$ ) and root-mean-square deviation (circles, RMS) on the degree of development of wind waves (a) and height differences $\Delta H_{S}$ (b) for the TOPEX/Poseidon radio altimeter (Karaev et al., 2002).

Figure 3 shows the obtained dependences for the mean values and root-mean-square deviations for scatterometers, and Fig. 4 shows the data for radio altimeters.

A strong dependence of the retrieved wind speed on the type of waves for both radar instruments is seen from the figures. The mean value is minimal if the fetch is short (the speed is underestimated compared to the actual values during development of wind waves) and increases as the wave height of arriving swell increases in the case of mixed waves for radio altimeters and scatterometers.

The situation is different from the point of view of variations in the backscattered radar cross section: the backscattered radar cross section decreases for radio altimeters and increases for scatterometers as the fetch increases (developing wind waves) or the wave height of the swell increases (mixed sea), which is seen in Fig. 5.

It is seen from Figs. 3 and 4 that development of wind waves is a uniform process; therefore, no significant variation in the RMS deviation is observed compared to the case of fully developed waves for both instruments.

The characteristic of arriving swell can vary in wide limits, which will influence the parameters of largescale waves. This is important first of all for radio altimeters when scattering is quasi-specular: the RMS deviation increases by more than a factor of two for large swell (see Fig. 4b) compared with the fully developed wind waves. 
(a)

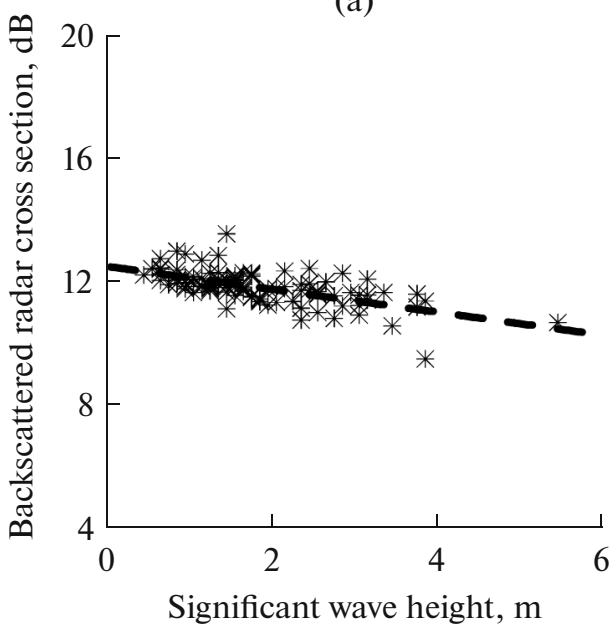

(b)

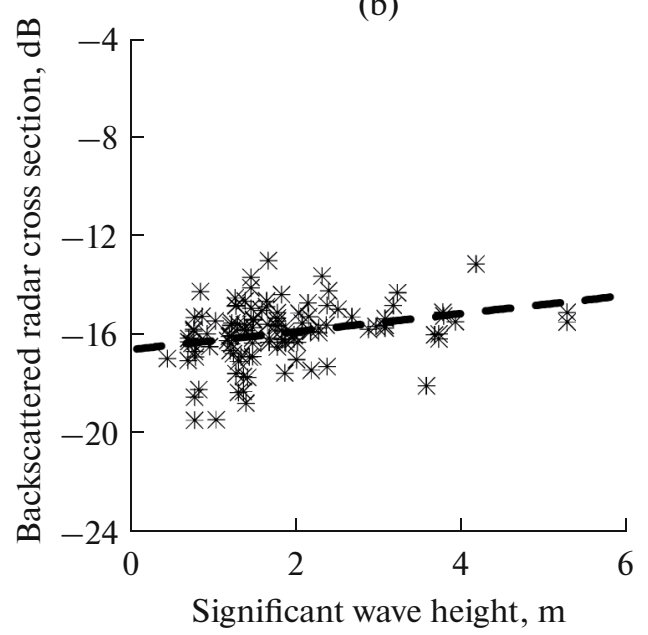

Fig. 5. Backscattered radar cross section vs. height of significant waves at fixed wind speed (in the interval $5.85-6.15 \mathrm{~m} / \mathrm{s}$ ) for the TOPEX/Poseidon radio altimeter (a) and ERS-1/2 scatterometer (b) (Karaev et al., 2002).

Resonance ripples, which depend on the local wind, are considered as the scattering object at medium incident angles. In this case, large-scale waves influence the intensity of backscattering by means of tilt modulation (variations in the local incident angle) and hydrodynamic modulation (variations in the spectral density of ripples along the profile of large-scale waves). This is a significantly weaker mechanism. Swell waves are long and gently sloping; therefore, they do not cause such a strong fluctuation of the backscattered radar cross section (of the retrieved wind speed) as during vertical sounding (see Figs. 3b and 4b).

Unfortunately, different states of the sea surface are not reliably distinguished on the "significant wave height-backscattered radar cross section" plane because the backscattered radar cross section actually depends on the variance of slopes but not on the wave height, as was mentioned before. However, the developed two-parameter algorithms allowed us to increase the accuracy of wind speed retrieval from the radioaltimeter data, although it is not possible to get rid of ambiguity completely.

The sea surface state has a strong influence on the backscattered radar cross section, which causes errors of wind retrieval using a one-parameter algorithm based on the scatterometer data. It is necessary to apply available information about the waves to increase the accuracy of retrieval.

\section{CONCLUSIONS}

The accuracy of surface wind retrieval from the radar data is not always satisfactory. This is caused by the fact that scattering occurs at the sea surface, which is formed not only under the influence of the local wind. The analysis of the radio-altimeter data showed that arrival of swell leads to a decrease in the backscattered radar cross section and consequently leads to overestimation of the retrieved wind speed (Karaev et al., 2006).

Since the mechanisms of backscattering at small and medium incident angles are different, it was interesting to repeat the analysis of the scatterometer data. In the analysis, we used the joint dataset containing scatterometer data (backscattered radar cross section, incident angle, direction of sounding) and the data of wave NDBC buoys (wind speed and direction, significant wave height).

Wind waves were divided into three types on the basis of the data of moored buoys on wind speed and significant wave height: developing waves, fully developed waves, and mixed sea. Data processing demonstrated that, similarly to the radio-altimeter data, the accuracy of wind speed retrieval is maximal for fully developed waves.

Fine spatial division was introduced for each type of waves. Data processing showed that, at the stage of wave development, wind speed is underestimated, while the arrival of swell leads to overestimation of the retrieved wind speed. The character of dependence coincides with the dependence obtained for radio altimeters.

The opposite situation is observed when we use the backscattered radar cross section: if the wave height increases, the backscattered radar cross section decreases for the radio-altimeter data and increases for the scatterometer data.

Thus, if we wish to increase the accuracy of surface wind speed retrieval, it is necessary to reject the application of the standard algorithm, take into account the type of waves, and use the corresponding processing algorithm. One of the versions is development of regional algorithms or application of additional information about the waves, for example, from the radioaltimeter data. 


\section{ACKNOWLEDGMENTS}

This work was supported by the Russian Foundation for Basic Research (grant no. 13-05-00852a and project no. 15-55-53046 GFEN_a); the Radiophysics Program of the Division of Physical Sciences, Russian Academy of Sciences; and the Chinese Academy of Sciences.

\section{REFERENCES}

Bass, F. and Fuks, I., Rasseyanie voln na statisticheski nerovnoi poverkhnosti (Wave Scattering from Statistically Rough Surfaces), Moscow: Nauka, 1972.

Davidan, I.N., Lopatukhin, L.I., and Rozhkov, V.A., Vetrovoe volnenie $v$ Mirovom okeane (Wind Waves in the World Ocean), Leningrad: Gidrometeoizdat, 1974.

Donelan, M. and Pierson, W., Radar scattering and equilibrium ranges in wind-generated waves with application to scatterometry, J. Geophys. Res., 1987, vol. 92, no. C5, pp. 4971-5029.

Glazman, R.E., Wind-fetch dependence of Seasat scatterometer measurements, Int. J. Remote Sens., 1987, vol. 8 , no. 11 , pp. 1641-1647.

Glazman, R.E. and Pilorz, S.H., Basic properties of developed wind waves and their influence on microwave remote sensing signatures, Proceedings of the 12th Canadian Symposium of Remote Sensing (IGARSS'89), 1989, no. 5, pp. 2980-2983.

Glazman, R. and Pilorz, S., Effects of sea maturity on satellite altimeter measurements, J. Geophys. Res., 1990, vol. 95, no. C3, pp. 2857-2870.

Gourrion, J., Vandemark, D., Bailey, S., Chapron, B., Gommenginger, G.P., Challenor, P.G., and Srokosz, M.A.,
A two-parameter wind speed algorithm for $\mathrm{Ku}$-band altimeters, J. Atmos. Oceanic Technol., 2002, vol. 19, no. 12 , pp. 2030-2048.

Guo, J., He, Y., Perrie, W., and Hui, S., Chu, X., A new model to estimate significant wave heights with ERS-1/2 scatterometer data, Chin. J. Oceanol. Limnol., 2009, vol. 27, no. 1, pp. 112-116.

Halpern, D., Satellites, Oceanography and Society, Halpern, D., Ed., Elsevier, 2000.

Karaev, V.Yu., Kanevsky, M.B., Balandina, G.N., Cotton, P.D., Challenor, P.G., Gommenginder, P., and Srocosz, M.A., On the problem of the near ocean surface wind speed retrieval by radar altimeter: Twoparametric algorithm, Int. J. Remote Sens., 2002, vol. 23, no. 16, pp. 3263-3283.

Karaev, V.Yu., Kanevskii, M.B., Balandina, G.N., and Gommenginger, C., Influence of regional features on the accuracy of determination of wind velocity over the ocean, Issled. Zemli Kosmosa, 2002, no. 4, pp. 32-42.

Karaev, V.Yu., Kanevskii, M.B., Meshkov, E.M., Cotton, D., and Gommenginger, C., Retrieval of the surface wind speed from satellite radio-altimeter data: A review of algorithms, Radiophys. Quantum Electron., 2006, vol. 49, no. 4, pp. 251-263.

Karaev, V.Yu., Meshkov, E.M., and Chu, X., Features of sea-wave classification in problems of remote sensing, Izv., Atmos. Ocean. Phys., 2013, vol. 49, no. 4, pp. 919929.

Valenzuela, G., Theories for the interaction of electromagnetic and oceanic waves: A review, Boundary Layer Meteorol., 1978, vol. 13, pp. 61-86.

Translated by E. Morozov 